\title{
Kernos
}

Revue internationale et pluridisciplinaire de religion grecque antique

$26 \mid 2013$

Varia

\section{Xénophon, l'oracle de Delphes et la divination}

\section{Louise Bruit Zaidman}

\section{OpenEdition \\ Journals}

\section{Édition électronique}

URL : http://journals.openedition.org/kernos/2200

DOI : $10.4000 /$ kernos.2200

ISSN : 2034-7871

\section{Éditeur}

Centre international d'étude de la religion grecque antique

\section{Édition imprimée}

Date de publication : 10 octobre 2013

Pagination : 59-72

ISSN : 0776-3824

\section{Référence électronique}

Louise Bruit Zaidman, "Xénophon, l'oracle de Delphes et la divination », Kernos [En ligne], 26 | 2013, mis en ligne le 31 octobre 2015, consulté le 02 mars 2021. URL : http://journals.openedition.org/ kernos/2200; DOI : https://doi.org/10.4000/kernos.2200 
Kernos 26 (2013), p. 59-72.

\section{Xénophon, l'oracle de Delphes et la divination}

Résumé : La pratique de la divination, telle que Xénophon l'expose tout au long de l'Anabase, est la mise en œuvre d'un mode de communication avec les dieux qui peut emprunter des formes diverses, mais dont la principale est le sacrifice et l'observation des signes positifs ou négatifs, offerts par les victimes. Elle suppose une bienveillance de la divinité toujours prête, semble-t-il, à répondre aux questions posées par les hommes, dans le domaine public comme dans le privé, pourvu que ces questions soient légitimes. Le reste de l'œuvre relève de la même conception, influencée par Socrate, comme le suggèrent les Mémorables, et trouve son origine dans la consultation de l'oracle de Delphes, au moment de son départ pour l'expédition organisée par Cyrus et qui deviendra l'expédition dite des Dix Mille.

Abstract: Divination, as described by Xenophon in the Anabasis, represents a means of communication with the gods, which can take several forms. The offer of a sacrifice is the first of these, with the observation of the signs given by the victims. This practice assumes that the gods are kind and that they are always ready to respond to questions posed by people either in public or in private, if these questions are legitimate. The same idea, influenced by Socrates, is found in all Xenophon's works, as evidenced in the Memorabilia, beginning with Xenophon's consultation of the Delphic oracle on joining Cyrus' expedition, which would known as the "Ten Thousand" expedition.

Xénophon est un auteur qui a subi, de la part de la critique moderne, les pires mépris et la pire condescendance en même temps que les suspicions les plus graves. L'auteur a été souvent accusé de mensonge, au mieux de mythomanie, son œuvre de faible et indigente, à l'image de sa pensée. Des études récentes ont entrepris de lui restituer une cohérence et une épaisseur qui en renouvellent l'intérêt. Mais de lourds soupçons continuent à peser sur la crédibilité qu'on peut accorder à ses propos. C'est principalement son œuvre apologétique qui appelle le soupçon, qu'il s'agisse du personnage qu'il dessine de lui-même dans l'Anabase ou du Socrate qu'il propose à travers les Mémorables ou ses autres écrits socratiques ${ }^{1}$. Ce n'est pas sur l'historicité de ses propos que je m'interrogerai aujourd'hui. Je me demanderai plutôt ce que peut nous apprendre sa façon d'aborder la question de

${ }^{1}$ Cf. le bilan dressé par L.A. Dorion, Introduction générale aux Mémorables de Xénophon, T.1, Paris, 2000 (CUF), p. XVIII-XCIX. Il renvoie en particulier à l'article de L. RoBIN, «Les Mémorables de Xénophon et notre connaissance de la philosophie de Socrate ", Année philosophique 21 (1910), p. 1-47 [repris dans La pensée hellénique, des origines à Epicure, Paris, 1942, p. 81-137], article qui contient « tous les principaux reproches formulés contre Xénophon ». Pour une lecture récente de Xénophon, cf. V. AZOULAY, Xénophon et les grâces du pouvoir. De la charis au charisme, Paris, 2004. 
la divination, une fois prise en compte la dimension apologétique d'une grande partie de son œuvre, et ses conséquences. Il nous offre en effet cette perspective particulière d'être à la fois observateur, en ce qui concerne l'art de la guerre en général et la pratique de la mantique en particulier, et praticien.

Xénophon se met en scène dans l'Anabase en tant que général et, devenu historien dans les Helléniques, il rend compte de la place et du rôle de la mantique dans les opérations qu'il décrit. Dans l'un et l'autre cas, c'est bien sûr en même temps son propre regard sur ce moyen particulier de communication avec les dieux qu'il met en scène, ce que confirment les ouvrages dans lesquels il théorise l'usage de ces pratiques, que ce soit à propos des grands hommes auxquels il consacre des traités à l'occasion de ses écrits sur l'art de la guerre, ou encore, à travers les propos ou comportements qu'il prête à Socrate notamment dans les Mémorables. Je voudrais montrer la cohérence de ces différents écrits, du point de vue qui nous occupe, et leurs interférences. Il apparaît en particulier que la pratique de Xénophon, approfondie et mise en œuvre au cours de l'expédition des Dix Mille, telle qu'il nous la présente, conditionne rétrospectivement, c'est-àdire au moment de la rédaction de l'Anabase, la place centrale qu'il accorde à la divination dans la relation aux dieux, et que cette pratique colore toute son approche ultérieure ${ }^{2}$. Surtout, Xénophon donne à sa consultation d'Apollon à Delphes, telle qu'il la présente au livre III de l'Anabase, un rôle qui en fait le moment dans lequel non seulement son avenir se joue, mais celui où se met en place pour lui un système d'interprétation dont Socrate occupe le centre. C'est du moins ainsi qu'il semble l'interpréter après coup et c'est ce que j'essaierai de vérifier. Que ses écrits reflètent la pratique de la divination en temps de guerre de l'époque classique, et que sa pratique soit marquée par l'influence spartiate et par son laconisme ${ }^{3}$, n'empêche pas que Xénophon la colore d'une façon particulière à travers sa propre expérience et sa propre conception du rapport aux dieux. C'est ce point, qu'éclaire l'importance que revêt chez lui la rencontre de Socrate, telle qu'il en rend compte.

\section{La consultation de l'oracle de Delphes}

Xénophon avait moins de trente ans lorsqu'il rencontra Socrate et que ce dernier l'invita à le suivre, selon l'anecdote rapportée par Diogène Laërce 4 .

2 Cf. R. PARKer, «One Man's Piety: The Religious Dimension of the Anabasis », in R. LANE Fox (éd.), The Long March. Xenophon and the Ten Thousand, New Haven/London, 2004, p. 131-153, spéc. p. 133 : «For Xenophon, one might say, Greek religion is above all a religion of divination. »

${ }^{3}$ Sur la pratique de la divination dans les guerres de l'époque classique, cf. W.K. PRITCHETT, The Greek State at War III, Religion, Berkeley, 1979, en particulier p. 47-90 : «The Military Mantike »; sur le laconisme de Xénophon dans ce domaine, R. PARKER, "Sacrifice and Battle », in H. VAN WEES (éd.), War and Violence in Ancient Greece, Londres, 2000, p. 299-314, et id., "Spartan Religion ", in A. Powell (éd.), Classical Sparta: Techniques bebind her success, Londres, 1989, p. 142-172.

4 Diogène Laërce, II, 48. 
C'était en 404, du moins selon l'hypothèse la plus souvent retenue ${ }^{5}$. Trois ans plus tard, Xénophon partait rejoindre son ami Proxène auprès de Cyrus le Jeune, cinq ans avant le procès et la mort de Socrate. Je voudrais montrer comment la conception de la divination qui se manifeste dans les diverses œuvres de Xénophon, qui ramassent et condensent l'expérience de toute sa vie; comment cette conception est profondément imprégnée par la marque que lui a laissée le philosophe, durant le bref temps pendant lequel il l'a fréquenté, et la façon dont Xénophon s'est forgé, à partir de lui, une image des rapports entre les dieux et les hommes.

Entre la consultation de l'oracle, qui va déterminer tout le reste de sa carrière militaire, et les Mémorables où il expose, vingt ans après, les convictions de Socrate telles qu'il les a comprises, il y a d'étroites correspondances, qui traduisent ce que Xénophon a tiré de sa fréquentation de Socrate et que reflètent à leur manière, me semble-t-il, sa pratique de stratège, son œuvre d'historien et ses traités théoriques, notamment sur la question qui nous intéresse ici.

La consultation de l'oracle de Delphes, telle que la rapporte Xénophon, scelle l'avenir immédiat du jeune aristocrate athénien et influence durablement sa vie. C'est au livre III de l'Anabase que Xénophon entre en scène, au moment de plus grand découragement de l'armée des mercenaires grecs, après la mort de Cyrus et la décimation des stratèges. On a souvent noté cette place centrale donnée à l'épisode et son rôle dans le dispositif apologétique de l'Anabase. C'est à ce moment que Xénophon se présente au lecteur en rappelant comment il s'est engagé dans l'expédition. C'est alors aussi qu'intervient Socrate auquel Xénophon a demandé conseil pour savoir s'il rejoindrait Proxène qui lui promet l'amitié de Cyrus :

Quand Xénophon eut lu sa lettre (celle de Proxène), il se concerta avec Socrate d'Athènes pour savoir s'il devait se mettre en route. Socrate, qui appréhendait que l'amitié avec Cyrus ne fût mal vue de la cité, parce que Cyrus passait pour avoir soutenu avec ardeur les Lacédémoniens dans leur guerre contre Athènes, conseilla à Xénophon d'aller à Delphes pour consulter le dieu sur ce voyage ${ }^{6}$.

La demande de conseil accrédite la proximité de Xénophon et de Socrate, et sa qualité de disciple du philosophe, qui ont parfois été contestées ${ }^{7}$. On a beaucoup glosé sur la façon dont Xénophon présente l'épisode et sur le rôle qu'il y a joué. On remarquera que l'initiative de consulter le dieu vient de

5 Dorion, o.c. (n. 1), p. XX-XXI, considère, contre l'avis notamment de P. Masqueray, (Xénophon: Anabase, livres I-III, introduction, texte et traduction, Paris, 1959) et de E. DeLEBECQue (Essai sur la vie de Xénophon, Paris, 1957), que «nous n'avons aucune donnée fiable» qui nous permette de l'affirmer.

${ }^{6}$ An. III, 1, 4. Les traductions de l'Anabase et des Mémorables sont empruntées à l'édition de la CUF.

${ }^{7}$ Entre autres par L. RoBIN, La pensée grecque et les origines de l'esprit scientifique, Paris, 1923, p. 188. 
Socrate et non de Xénophon et aussi que la formule choisie est très générale (« consulter le dieu ») et ne préjuge pas de la question à poser.

Xénophon y alla. Il demanda à Apollon à quel dieu il devait offrir des sacrifices et des prières, pour parcourir dans les meilleures conditions la route à laquelle il songeait et pour revenir sain et sauf, après un plein succès. Apollon lui indiqua les dieux auxquels il fallait qu'il sacrifiât.

On a glosé tantôt sur l'outrecuidance, tantôt sur la duplicité de Xénophon et de la question posée. Anecdote peu glorieuse, lit-on ailleurs ${ }^{8}$. Socrate quant à lui, reproche à Xénophon d'avoir «décidé tout seul qu’il devait faire ce voyage ». C’est-à-dire d'avoir anticipé la réponse du dieu. Ce qu'il lui reproche est plutôt une imprudence de jeune homme qu'une impiété. Or cette décision imprudente ou audacieuse va décider de toute sa vie. Il reste en effet que le dieu répond et, par là, implicitement, accepte la question dans la forme que lui a donnée Xénophon. Cette réponse, il convient de s’y soumettre, comme le fait observer Socrate, ce que fait Xénophon en sacrifiant aux divinités indiquées.

Cet épisode est emblématique du rôle que Xénophon prête à l'oracle, et de la médiation de Socrate ${ }^{9}$. Il faudra attendre le livre VI pour apprendre que Zeus-roi était le dieu qui lui avait été désigné à Delphes ${ }^{10}$. Apollon a donc répondu à sa question, et dans le sens souhaité. Xénophon reviendra effectivement de son expédition sain et sauf avec un plein succès, si l'on veut bien tenir pour crédible sa carrière de stratège dans l'armée des Dix Mille et les conditions dans lesquelles s'achève le récit de l'Anabase. À Lampsaque, Xénophon rencontre le devin Euclide de Phlionte qui se réjouit avec lui qu'il eût la vie sauve ${ }^{11}$. Voici accomplie la première promesse du dieu. Mais Xénophon lui avoue son dénuement : il n'a pas même de quoi rentrer à Athènes. C'est dans les entrailles d'une victime offerte à Apollon encore, que le devin découvre l'hostilité de Zeus Meilichios ${ }^{12}$, auquel Xénophon a négligé de sacrifier depuis son départ d'Athènes, et c'est à la suite de l'holocauste que Xénophon offre à ce dieu sur le conseil du devin, que la

8 Dorion, o.c. (n. 1), p. XXIX : «Pour l'homme pieux qu'il prétend être, il semble plutôt indigne non seulement d'avoir rusé avec la divinité et avec Socrate, mais aussi, mais surtout, de s'en flatter. »

${ }^{9}$ Cet épisode souvent commenté, montre-t-il un Xénophon « candide» (PARKER, l.c. [n. 2], p. 147) ou faut-il considérer qu'ici «il se moque de nous », selon la conviction de P. Bonnechere (discussions lors du Colloque) ? On peut considérer que Xénophon nous «balade» et que l'épisode est «fabriqué » pour le mettre mieux en valeur, on peut aussi envisager que, malgré la part de reconstruction dans la présentation des événements et leur succession, le récit reste significatif d'un mode de fonctionnement dans le rapport au divin qui nous renseigne, au-delà de Xénophon lui-même, sur les pratiques et la pensée religieuse de ses contemporains. Et ceci vaut pour l'ensemble de son récit.

10 An. VI, 1, 22.

11 An. VII, 8, 1-6.

12 Cf. J.-M. PAIller, «Zeus ex machina. Le Meilichios ou l'oubli de la troisième fonction », Pallas 85 (2011), p. 43-57, spéc. p. 43-45 (La femme, la parenté et le politique. Hommage à Claudine Leduc). 
fortune va tourner pour lui comme l'ont annoncé des victimes favorables

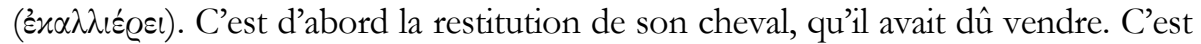
ensuite à Pergame où Xénophon remet l'armée à Thibron, après être allé saluer le dieu $(\hat{\eta} \sigma \pi \dot{\alpha} \sigma \alpha \tau o)$ :

Les Laconiens en effet, les lochages, les autres stratèges et les soldats étaient d'accord pour qu'il fit son choix parmi les chevaux, les pairs de bœufs et le reste du butin, si bien que dès lors il fut en état d'obliger même autrui.

Ainsi s'accomplit, semble-t-il, la deuxième partie de l'oracle. L'interprétation suggérée par le récit de Xénophon alimente le scepticisme de nombreux commentateurs. Pourtant, ne faut-il pas envisager que pour le «croyant», il y a toujours matière à justifier sa « foi »?...

\section{Socrate et la divination - les Mémorables}

À la mise en scène vivante et au dialogue entre Socrate et le jeune Xénophon, font écho les propos par lesquels, dans les Mémorables, le même Xénophon définit la conception socratique de l'usage de la divination. Les Mémorables se présentent chez Diogène Laërce comme les notes prises par Xénophon à l'écoute de Socrate. Hypothèse bien fragile et fortement contestée ${ }^{13}$. La part de reconstitution, voire de fiction sont à prendre en compte, ainsi que la perspective apologétique qui est celle de Xénophon, comme dans l'Anabase. De fait, les propos prêtés à Socrate, et le portrait qu'ils dessinent du philosophe, nous éclairent autant ou plus sur Xénophon que sur son maître et c'est ce qui nous intéressera ici. Je laisserai donc de côté les débats sur l'historicité du Socrate de Xénophon pour m'arrêter à ce que ce dernier lui fait dire de la divination et y confronter l'écho de ces propos dans l'œuvre de son disciple.

Dans leur souci de laver Socrate des accusations portées contre lui, les Mémorables donnent à la divination une place centrale dans la pratique et la philosophie du maitre. Le recours à la divination ( $\mu \alpha \nu \tau \iota x \tilde{n} \chi \varrho \omega ́ \mu \varepsilon v o \varsigma)$, est introduit aussitôt après la mention de la pratique du sacrifice et donne lieu à un long développement où la pratique publique de Socrate est détaillée ainsi que ses propos sur cette question : "On le voyait souvent faire des sacrifices à la maison de même que sur les autels publics de la cité, et ce n'était pas non plus un secret qu'il avait recours à la divination $»^{14}$. Le rôle central du daimonion, qui a conduit à l'accuser d'introduire de nouveaux dieux, est mis en perspective de ce point de vue :

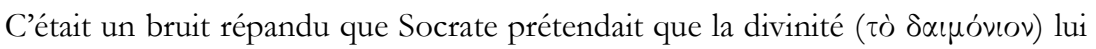

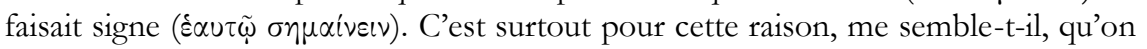
l'a accusé d'introduire des divinités nouvelles.

\footnotetext{
${ }^{13}$ DORION, o.c. (n. 1), p. XXXVI-XXXIX.

${ }^{14}$ Mém. I, 1, 2.
} 
Toute l'argumentation de Xénophon pour disculper son maître sur ce point, repose sur l'assimilation de ce signe de la divinité à ce que chacun, à Athènes, appelle divination, comme l'a montré L.A. Dorion ${ }^{15}$ : «Il n'a rien introduit de plus nouveau que les autres, ceux qui croient à la divination et font appel aux oiseaux, aux voix, aux coïncidences, et aux sacrifices ». Socrate a fait bénéficier un grand nombre de ses compagnons de ce que la divinité lui avait signifié à l'avance

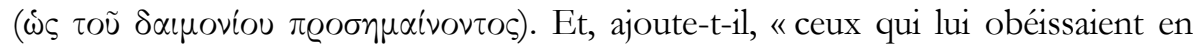
tiraient profit, ceux qui ne lui obéissaient pas s'en mordaient les doigts $»^{16}$. Le conseil de Socrate à Xénophon de consulter le dieu, et son reproche à son retour de Delphes, assorti d'une invitation à obéir au dieu, viennent ainsi prendre place dans le cadre d'un comportement habituel du philosophe, qui met à la fois en lumière son rôle bénéfique vis-à-vis de ses compagnons et sa piété sans reproche. Il reste alors à Xénophon à préciser, avec Socrate, le contexte dans lequel les hommes doivent avoir recours à la divination. Car,

on a besoin de recourir à la divination pour bien administrer les maisons et les cités lorsque l'issue est incertaine. Dans ce cas, Socrate envoyait ses amis consulter les oracles. Mais le sage doit savoir distinguer entre les choses qui dépendent des dieux et celles qui relèvent de l'art ou de la connaissance humaine ${ }^{17}$.

Entre la conception socratique de la divination présentée dans les Mémorables, et la pratique du stratège et de l'observateur des campagnes guerrières, il y a une intime relation. On en trouve une confirmation dans la conclusion de l'Économique. Mais c'est l'ensemble des activités humaines qui relèvent de proche en proche, de la consultation des dieux. À Critobule qui vient d'évoquer les accidents qui menacent l'agriculture, Socrate répond :

La puissance des dieux ne s'étend pas moins, Critobule, sur l'agriculture que sur l'art militaire. Ne vois-tu pas qu'avant de commencer une action, l'homme se les rend propices, qu'il les consulte sur ce qu'il doit faire ou ne pas faire en interrogeant les entrailles des victimes ou le chant des oiseaux?

Finalement l'histoire de la consultation de l'oracle de Delphes est plus qu'une anecdote. Elle met en scène un Socrate conforme à celui que dessinent les œuvres dites socratiques de Xénophon, Apologie, Banquet, Mémorables, Économique; elles montrent en face de lui un jeune homme ardent et désireux d'aventure, mais qui se soumet aux conseils du maitre et aux injonctions de l'oracle. Sa question, si elle montre sa résolution, n'est pas plus inconvenante que celle de ce consultant de Dodone qui demande : «Auquel des dieux adresser sa prière pour réussir ce qu'il a dans l'esprit ? ». Il ne s'agit pas de piéger le dieu, ou de « ruser » avec lui, mais plutôt de négocier avec lui. Les sacrifices répétés auxquels on assiste dans le

15 L.-A. DORION, «Socrate, le daimonion et la divination », in J. LAURENT (éd.), Les dieux de Platon, Presses Universitaires de Caen, 2003, p. 169-192, spéc. p. 171-172.

${ }^{16}$ Mém. I, 1, 2-4.

17 Mém. I, 1, 6. 
contexte militaire en cas de réponse non favorable ne sont-ils pas une autre forme de négociation?

La narration que Xénophon propose dans l'Anabase, est à la fois le récit d'événements vécus tels qu'ils les a mémorisés ou qu'il les reconstitue à distance, et l'expression de la conception de la relation aux dieux qui est la sienne, notamment à travers la pratique de la divination, telle que nous l'avons vue définie par le Socrate de Xénophon. C'est à cette narration que je vais venir maintenant.

\section{Du bon usage des devins}

Je ne reviendrai pas ici sur ce que R. Parker a appelé «the Xenophontic system » et que je tiens pour acquis. Xénophon est en effet de loin la source la plus riche sur les différentes formes du sacrifice en temps de guerre. C'est notamment à partir de Xénophon que R. Parker définit six contextes de sacrifices " consultatifs », c'est-à-dire relevant de la divination ${ }^{18}$.

Dans l'Anabase, la présence des devins dans l'armée, préposés à l'interprétation des réponses des dieux données par l'intermédiaire des sacrifices, est fréquemment évoquée ${ }^{19}$. Parfois ils sont mentionnés de manière anonyme, ainsi ils s'opposent collectivement aux stratèges qui ont prévu de faire du butin sur le pays des Tibarènes qui leur ont pourtant envoyé des présents d'hospitalité : "Après avoir immolé force victimes, à la fin, les devins émirent tous l'avis que les dieux n'approuvaient en aucune façon qu'on attaquât ces gens-là »20. Au chapitre $\mathrm{V}$ « un des devins $(\tau \tilde{\omega} \nu \mu \alpha \dot{\alpha} \nu \tau \varepsilon \omega \nu \tau \iota \varsigma) »$, pour répondre à une situation dans laquelle les hommes sont épuisés et gelés par une étape face au Borée conseille de sacrifier au vent; le sacrifice a lieu « et tous virent très nettement que le souffle de l'air avait diminué de violence ». Mais le sacrifice ne règle pas tout : il n'empêche pas que la neige a six pieds d'épaisseur «si bien qu'un grand nombre de bêtes de somme, d'esclaves succombèrent et une trentaine de soldats ». Dans le cas du passage du Centritès, les devins accomplissent les sacrifices tandis que les stratèges avancent à la tête de leurs troupes. Très souvent, ils assistent le stratège qui accomplit le sacrifice. Tantôt encore c'est le stratège lui-même qui fournit une interprétation, fort d'une science acquise par l'expérience, comme Xénophon lui-même, à l'occasion, le précise devant les soldats, pour contrer les attaques du devin Silanos : «Il savait bien qu'en ces sortes de choses (la lecture des entrailles) je ne suis pas sans expérience, parce que continuellement j'assiste aux sacrifices $»^{21}$. C'est dans le même esprit que Cyrus, dans la Cyropédie soutiendra que les chefs

18 R. PARKer, l.c. (2000, n. 3), p. 299-314. Voir aussi M.H. JAMESON, «Sacrifice before Battle », in V.D. HAnson (éd.), Hoplites: The Classical Greek Battle Experience, Londres, 1991, p. 197-227.

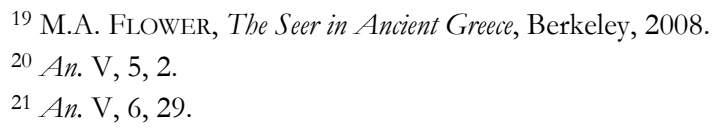


doivent savoir eux-mêmes interpréter les signes divins afin de ne pas être à la discrétion des devins, dans le cas où ceux-ci voudraient le tromper ${ }^{22}$.

Quelques devins sont désignés par leur nom et leur lieu d'origine; c'est le cas pour des devins que la réputation, le rôle ou le prestige distinguent des autres ${ }^{23}$; c'est le cas d'Arexion, il vient de Parrhasie en Arcadie qui semble avoir produit plusieurs devins réputés. Le contingent Arcadien est par ailleurs particulièrement important puisque Xénophon précise qu'avec les Achéens ils forment plus de la moitié de l'armée ${ }^{24}$. L'Arcadien Arexion apparaît à plusieurs reprises, notamment au port de Calpée, moment difficile car la nourriture manque, l'armée est bloquée dans l'attente de bateaux et les Thraces les menacent. Mais les sacrifices n'autorisent pas au départ :

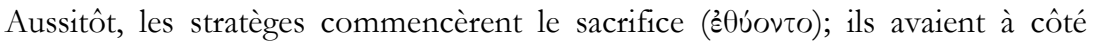
d'eux le devin Arexion d'Arcadie. Les victimes immolées pour le départ n'étaient

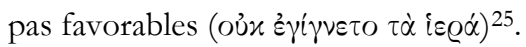

La densité des sacrifices augmente avec les situations d'aporie. Le lendemain de ce premier échec, le stratège « recommence jusqu’à trois fois » pour savoir si l'on peut partir et les signes donnés par les victimes restent contraires. Les questions posées aux dieux varient avec la situation. On leur demande d'abord si on peut se mettre en route, ensuite, si on peut aller aux vivres, mais, là encore, " on sacrifia encore jusqu'à trois fois, et les victimes furent défavo-

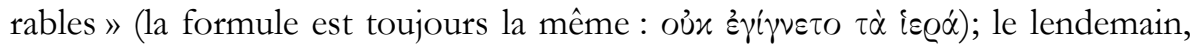
on sacrifie à nouveau, et Xénophon cède sa place au devin Cléanor d'Arcadie, mais le résultat est à nouveau négatif. Pour finir, devant la disette générale (on n'a même plus d'animaux de sacrifice et il faut immoler des bœufs d'attelage), le stratège Néon brave les présages contraires et sort avec deux mille hommes pour aller aux vivres. Attaqués par les cavaliers de Pharnabaze ils sont taillés en pièce. À cette nouvelle, Xénophon égorge un bœuf d'attelage ( $\sigma \varphi \alpha \gamma \iota \alpha \sigma \alpha \dot{\alpha} \mu \varepsilon v o \varsigma)$ et part au secours des survivants. C'est seulement le lendemain, après une nuit passée sous les armes, alors que Xénophon sacrifie, " pour savoir si on pouvait

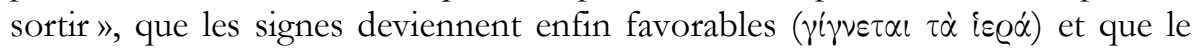
devin Arexion qui l'assiste, aperçoit un aigle à droite et engage Xénophon à se mettre à la tête des troupes. Le même devin intervient encore face aux cavaliers et fantassins envoyés par Pharnabaze. Alors que les deux armées sont face à face, il égorge une victime $(\sigma \varphi \alpha \gamma \iota \alpha \zeta \varepsilon \tau \alpha \iota)$ et, cette fois, « du premier coup les

22 Cyropédie I, 6, 2.

${ }^{23}$ Cf. H. Bowden, "Oracles for Sale», in P. Derow, R. PArker (éd.), Herodotus and his World, Oxford, 2003, p. 252-274, spéc. p. 270-274.

24 An. VI, 2, 10.

25 An. VI, 4, 13. 


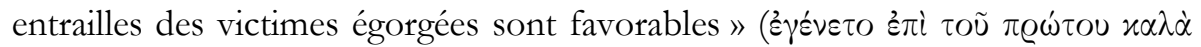
$\tau \dot{\alpha} \sigma \varphi \dot{\alpha} \gamma(\alpha)^{26}$.

Un seul devin est présenté sous un jour négatif. Il s'agit de Silanos d'Ambracie, qui s'est mis d'abord au service de Cyrus, ce qui lui a valu une forte récompense. C'est soucieux de préserver son butin qu'il interviendra pour pousser au départ, contre Xénophon qui prône, avant tout, l'unité, mais auquel le devin reproche d'envisager de créer une colonie où se fixer, ce en quoi il n'a peut-être pas tort ${ }^{27}$. Il n'en reste pas moins qu'on apprendra un peu plus tard qu'il s'est sauvé, sur un bateau qu'il avait affrêté ${ }^{28}$. Toujours prêt à soulever les soldats contre leur stratège dans les situations difficiles, Silanos n'est nullement représentatif et montre seulement comment, parmi les devins peuvent se glisser des individus intéressés et sans courage.

\section{Les pratiques divinatoires au service du stratège}

Mais loin que le recours aux sacrifices soit le seul moyen de communiquer avec les dieux, tout l'éventail des pratiques divinatoires, tel que le résume la formule des Mémorables s'étale au long du récit: la divination fait appel aux oiseaux, aux voix, aux coïncidences et aux sacrifices ${ }^{29}$. C'est un écho de cette formule que répètent la plupart des œuvres de Xénophon. Ainsi le Grand Cyrus au moment de mourir :

Ô Zeus ancestral, Soleil et tous les dieux ! Acceptez ces offrandes en action de grâce pour toutes les œuvres louables que je vous dois et ces autres actions de grâce pour m'avoir montré par des présages, par des signes célestes, des oiseaux et des voix ce qu'il fallait faire et ne pas faire... ${ }^{30}$

Je ne prendrai qu'un exemple dans l'Anabase de l'application de ces principes à la pratique militaire de Xénophon.

Le livre IV commence par le récit de la traversée du pays des Cardouques, alors que les mercenaires grecs, sous la conduite de Chirisophe, remontent vers les sources du Tigre et de l'Euphrate, n'ayant pas réussi à franchir le Tigre en aval. La situation est menaçante. Xénophon dans la descente de la montagne, a été

26 An. VI, 5, 8. Sur ce type de sacrifice accompli par égorgement sur le front de l'armée, aussitôt avant l'engagement, voir J.-P. VERNANT, «Artémis et le sacrifice préliminaire au combat», REG 101 (1990), p. 230-239; Figures, idoles, masques, Paris, 1990 : "Artémis et la guerre », p. 169-178, un sacrifice contre 'la menace d'ensauvagement' contenue dans la guerre. Une analyse différente chez JAMESON 1991, et PARKER 2000 (l.c. supra n. 17).

27 An. V, 6, 34. Cf. J. Dillery, « Chresmologoi and Manteis. Independant Diviners and the Problem of Authority ", in S.I. Johnston, P. STRUCK (éd.), Mantike. Studies in Ancient Divination, Leiden, 2005, p. 167-231, spéc. p. 198.

$$
\begin{aligned}
& { }^{28} \text { An. VI, 4, } 13 . \\
& { }^{29} \text { Mém. I, 3. } \\
& { }^{30} \text { Cyr. VIII, 7, } 3 .
\end{aligned}
$$


abandonné par l'homme qui portait son bouclier et est secouru de justesse par un hoplite, dont il donne le nom, et qui l'abrite sous son propre bouclier ${ }^{31}$. Arrivés sur la rive gauche du Centritès, les Grecs bivouaquent dans l'angoisse du lendemain. C'est alors que Xénophon a un songe (óv $\alpha Q)$ : » il lui semblait qu'il était lié par des entraves et que ces entraves tombaient d'elles-mêmes autour de lui; alors il était libre et pouvait marcher autant qu'il voulait $»^{32}$. Xénophon interprète aussitôt le rêve comme un signe favorable envoyé par les dieux. Le songe est confirmé par un sacrifice auquel assistent tous les stratèges et dont les signes sont

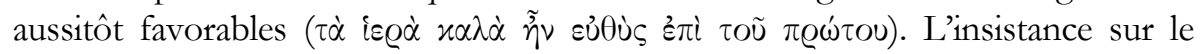
caractère immédiat et sans hésitation du résultat se comprend aisément si on met en regard toutes les occasions dans lesquelles il faut répéter l'opération, parfois plusieurs jours de suite. Ces signes indiscutables offerts aux Grecs par l'observa-

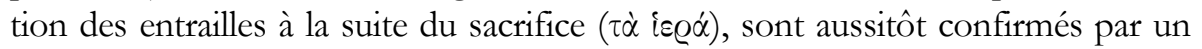

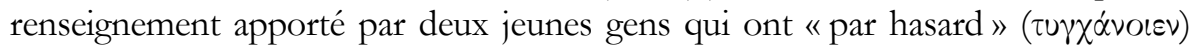
trouvé un gué. Le commentaire qui suit est éclairant :

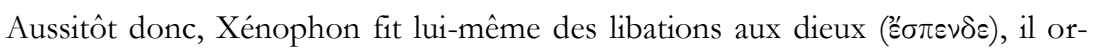
donna de verser du vin aux jeunes gens, et de prier les divinités qui lui avaient envoyé ce songe et découvert ce passage, pour qu'elles menassent aussi le reste à bien.

Les grâces rendues aux dieux (les libations) et les prières, les engagent en même temps qu'elles sont un signe public à l'intention des mercenaires qui ont le plus grand besoin d'encouragement avant l'action qui se prépare. La prière fait le lien entre les deux incidents, l'un privé, le songe, jusqu'à ce que Xénophon le révèle, l'autre qui pourrait paraître anecdotique, la découverte fortuite des jeunes gens; les deux incidents sont institués " manifestations divines » par le commentaire de Xénophon. Ainsi est dûment préparé le passage du fleuve par l'armée alors que la cavalerie ennemie les attend sur l'autre rive. Chirisophe donne le signal, une couronne sur la tête et la colonne se met en route.

Pendant ce temps les devins égorgeaient les victimes sur le Centritès (oi $\mu \dot{\alpha} v \tau \varepsilon ı \varsigma$

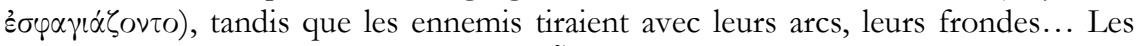
sacrifices étant favorables (

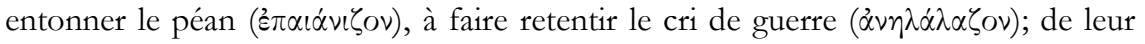
côté les femmes poussaient toutes des cris aigus ( $\sigma \nu v \omega \lambda o ́ \lambda u \zeta o v)$, car nombreuses étaient dans l'armée les hétaïres.

Dans ce superbe tableau de genre à sujet militaire, digne des grandes compositions de notre XIX ${ }^{\mathrm{e}}$ siècle, on voit admirablement comment la communication avec les dieux peut prendre les formes les plus diverses, du point de vue de la variété des signes donnés par les dieux (ici : songe, signe fortuit, entrailles favorables), qui peuvent être sollicités (par exemple par le sacrifice) ou non; mais aussi

\footnotetext{
31 An. IV, 2, 20.

32 An. IV, $3,8$.
} 
que cette relation est à double sens et a besoin d'être entretenue et encouragée. Les hommes, et d'abord les stratèges, ne cessent de manifester qu'ils ont bien reçu les signes et qu'ils en sont reconnaissants, une manière d'encourager les dieux à continuer de les soutenir. Et les dieux ici sont désignés par un pluriel collectif qui les engage tous, tous ceux au moins qui sont concernés par le combat. Ainsi, le comportement de Xénophon, s'il lui donne évidemment le beau rôle, n'en est pas moins conforme à ce qu'on attend d'un stratège digne de ce nom : montrer sa capacité à ajuster son interprétation aux signes observés, et son action à son interprétation. On remarquera l'intervention rarissime de l'élément féminin, dont Xénophon a besoin de préciser la présence dans l'armée, tellement il en a peu été question. Leur intervention confirme qu'on est bien à ce moment dans une de ces situations extrêmes qui justifient l'engagement de toutes les parties concernées et menacées dans leur survie.

Les Helléniques mettent en place le même système interprétatif. Je ne reviendrai pas sur le rapport d'Agésipolis, le roi spartiate, aux différents procédés divinatoires dans sa guerre contre Argos. L'épisode a été maintes fois analysé ${ }^{33}$. Je reprendrai par contre l'histoire des Phliasiens auxquels Xénophon consacre un long excursus (VII, 2, 1-23) en le justifiant par la qualité des belles actions accomplies par cette petite cité.

En butte aux attaques répétées des Argiens et des Arcadiens après la défaite de Leuctres, les Phliasiens finissent par demander de l'aide à Charès, le stratège athénien, pour escorter un convoi de vivres destiné à leur cité assiégée. Ils commencent par lui conseiller de «se mettre en communication avec les dieux

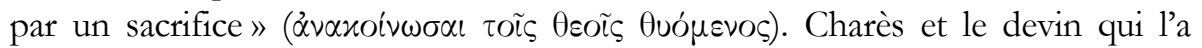
assisté, viennent au devant des Phliasiens en leur annonçant que «les signes sont favorables » ( $\varkappa \alpha \lambda \dot{\alpha} \tau \dot{\alpha} i \varepsilon \varrho \alpha ́)$. Dès que le héraut eût donné le signal, c'est comme si un dieu avait inspiré de l'ardeur aux mercenaires pour sortir en hâte ». Le succès est total, l'ennemi s'enfuit en abandonnant ses vivres dont les soldats vainqueurs font leur repas, non sans offrir auparavant des libations d'action de grâce ni sans chanter le Péan. Le succès à son tour, provoque une sorte de contagion : dans la foulée, les Corinthiens assurent des convois de vivre pour aider à la construction du rempart. Le plein succès de l'opération est alors célébré selon le rituel attendu après une victoire complète. La célébration des Phliasiens par Xénophon s'étend au comportement religieux de leurs chefs qui a mis en branle le processus en indiquant la conduite à tenir : commencer par entrer en communication avec les dieux qui, à leur tour, ont apporté leur appui à la vaillance et au courage.

33 Cf. notamment H. BOWDEN, "Xenophon and the scientific study of religion », in C.J. Tuplin (éd.), Xenophon and his world, Stuttgart, 2004, p. 236-237. Cf. aussi L. Bruit Zaidman, Le commerce des dieux, Paris, 2001, p. 67-69. Sur les pratiques religieuses dans les Helléniques, V. Gray, The Character of Xenophon's Hellenica, Londres, 1989, contra C.J. Tuplin, The Failings of Empire. A Reading of Xenophon Hellenica 2.3.11-7.5.27, Stuttgart, 1993. On peut voir aussi L. BRUIT ZAIDMAN, "Des pratiques et des dieux dans les Helléniques de Xénophon », in Xénophon, Cabiers des études anciennes 45 (2008) [V. AzOULAY éd.]. 
La divination est donc le mode privilégié de relation avec les dieux, avec le sacrifice auquel elle est intimement liée. De ce point de vue, la consultation des oracles et la lecture des entrailles relèvent de la même démarche intentionnelle, quand les signes «spontanés » appellent une interprétation sans qu'il y ait eu sacrifice préalable. Mais les différents types de divination interfèrent et se complètent l'un l'autre.

\section{Divination et piété}

La piété de Xénophon, dont nous ne nous demanderons pas ici, si elle est «traditionnelle », voire «conformiste » ou pas : vieux débat, qui nous intéresse moins que la question de savoir quelle représentation cette piété suppose de la relation des hommes aux dieux et de ses médiations possibles; cette piété, réside, en dernière analyse, dans le rapport que Xénophon établit entre les dieux et sa propre action, dans toutes les circonstances, et à tous les moments de sa vie. L'idée de départ est que la vie des hommes se passe tout entière sous le regard des dieux ${ }^{34}$ :

De fait, il croyait que les dieux se soucient des hommes, mais pas de la façon dont se l'imaginent la plupart des gens. Ceux-ci croient en effet que les dieux savent certaines choses et qu'ils en ignorent d'autres; Socrate croyait au contraire que les dieux savent tout (aussi bien les paroles et les actions que les projets que l'on tait); qu'ils sont présents partout, et qu'ils font signe aux hommes relativement à toutes les affaires humaines.

Citons encore la conclusion de l'entretien avec Aristodème :

Honore donc les dieux, tu sauras alors s'ils veulent t'éclairer sur ce qu'ils ont caché à la raison des hommes; tu reconnaîtras alors que telle est la grandeur de la divinité, qu'elle voit tout d'un seul regard, qu'elle entend tout, qu'elle est partout, qu'elle porte en même temps tous ses soins sur toutes les parties de l'univers ${ }^{35}$.

Cette conception justifie la place que Socrate et Xénophon avec lui, attribuent à la consultation des dieux dans toutes les actions entreprises. Pour Xénophon, tout se passe comme si l'impulsion première avait été le conseil de Socrate d'aller consulter l'oracle de Delphes. Le recours à la divination sous toutes ses formes dans les circonstances difficiles de l'expédition des Dix Mille, s'accompagne d'une interprétation permanente des moindres signes donnés par la divinité. Il vit «sous le regard des dieux». Non seulement «le monde est pour lui plein de dieux», mais ces dieux ont le regard posé sur les hommes

\footnotetext{
${ }^{34}$ Mém. I, 1, 19.

35 Mém. I, 4.
} 
pour répondre à leur questionnement et les guider à leur demande ${ }^{36}$. S’ils négligent leurs avis, ils ne devront s'en prendre qu'à eux des conséquences néfastes de leurs actes. Mais pour les hommes pieux, c'est à dire attentifs à leurs avis, l'espoir est permis de connaître la réussite de leurs projets et une vie digne d'être louée; Ce sont les recommandations de Socrate dans le dernier chapitre des Mémorables :

Si quelqu'un voulait s'élever au-dessus des connaissances humaines, il lui conseillait de s'adonner à la divination, lui assurant que, quand on sait par quels signes les dieux font connaitre leur volonté à l'homme, on n'est jamais privé des avertissements des dieux ${ }^{37}$.

\section{C'est encore le propos de l'Hipparque:}

Or, en l'occurrence, il est impossible de trouver à qui demander conseil sinon aux dieux : ils savent tout et avertissent selon leur bon vouloir, au moyen des signes des victimes, des oiseaux, des oracles et des songes. Et il est naturel qu'ils veuillent bien donner leurs avis à ceux qui ne se bornent pas à les interroger sur la conduite à tenir dans les moments critiques, mais qui, jusque dans les heures fortunées servent de toutes leurs forces la divinité ${ }^{38}$.

La conclusion des Mémorables, hommage de Xénophon à son maître, est une autre confirmation de la façon dont il s'applique les préceptes recommandés par ce dernier :

Pour moi qui l'ai vu tel que je l'ai dépeint, si pieux qu'il ne faisait rien sans l'assentiment des dieux, ... si juste qu'il rendit les plus grands services à ceux qui le fréquentaient, si prudent qu'il ne se trompait jamais dans l'appréciation du bien et du mal... habile à juger les gens, à leur montrer leurs fautes et à les tourner vers la vertu et vers le bien ${ }^{39}$.

Xénophon n'est pas un individu «confit en dévotion», il est un homme d'action. Mais peut-être faut-il prendre au sérieux la conviction qu'il affiche qu'il faut dans toute activité humaine, commencer, comme le commandant de cavalerie de l'Hipparque, par "offrir un sacrifice et s'assurer la faveur des dieux ${ }^{40}$ », même lorsqu'il s'agit de choisir un ami comme l'affirme le Socrate des Mémorables.

Le Xénophon de l'Anabase prend place dans la série des portraits de chef idéal qui s'esquisse au fil de son œuvre ${ }^{41}$. Sa pratique religieuse, dans laquelle son recours à la divination occupe une place primordiale, est un élément non

\footnotetext{
36 Sur la bienveillance attribuée aux dieux Grecs, cf. R. PARKER, «Gods Cruel and Kind: Tragic and Civic Theology ", in C. Pelding (éd.), Greek Tragedy and the Historians, Oxford, 1997, p. 143-160. Cf. aussi, R. PARKER, l.c. (n. 2, 2004), p. 131-153.

${ }^{37}$ Mém. IV , 7, 10.

${ }^{38}$ Hipparque, 9, 9.

${ }^{39}$ Mém. IV, 8, 11.

${ }^{40}$ Hipp., 2, 4.

${ }^{41}$ Cf. J. Luccioni, Xénophon et le socratisme, Paris, 1953, p. 108 et 143, et Azoulay, o.c. (n. 1).
} 
négligeable de cette construction. Le récit que Xénophon propose dans l'Anabase de ses différentes interventions dans ce domaine, fait l'objet, comme le reste, d'une mise en scène et d'une rhétorique méditées. Mais c'est l'ensemble de son œuvre qui porte témoignage d'une conception des rapports entre les hommes et les dieux fondée sur la conviction que les dieux sont toujours prêts à répondre aux sollicitations des hommes pourvu qu'elles prennent les formes attendues et qu'elles portent sur des questions qui méritent leur intervention. Ce sont les fautes des hommes et des cités qui entraînent les uns et les autres à leur perte malgré les signes divins ${ }^{42}$.

Louise BRUIT ZAIDMAN

ANHiMA UMR 8210

Université Paris-Diderot / Paris 7

Courriel:bruit@ehess.fr

42 Oracles et manifestations divines se multiplient à la veille de Leuctres pour dissuader en vain les Spartiates d'engager la bataille (Hell. VI, 4, 7). 